\title{
Existence and Stability of the Periodic Orbits Induced by Grazing Bifurcation in a Cantilever Beam System with Single Rigid Impacting Constraint
}

\author{
Run Liu ${ }^{a}$, Yuan Yue ${ }^{b, *}$, Jianhua Xie \\ ${ }^{\mathrm{a}, \mathrm{b}}$ Applied Mechanics and Structure Safety Key Laboratory of Sichuan Province, School of Mechanics and \\ Engineering, Southwest Jiaotong University, Chengdu 610031, China \\ ${ }^{\mathrm{e}}$ School of Mechanics and Engineering, Southwest Jiaotong University, Chengdu 610031, China
}

Abstract

Grazing which can induce many nonclassical bifurcations, is a special dynamic phenomenon in some non-smooth dynamical systems such as vibro-impact systems with clearance. In this paper, the existence and stability of the periodic orbits induced by the grazing bifurcation in a cantilever beam system with impacts are uncovered. Firstly, the Poincaré mapping of the system is obtained by the discontinuous mapping method. Secondly, the periodic orbits are determined by means of shooting method, and Jacobian matrix in the case of non-impact is obtained subsequently. Thirdly, for various impacting patterns, a combination of inhomogeneous equations and inequations is obtained to determine the existence of period orbits after grazing. Furthermore, the stability criterion of the grazing-induced periodic orbits is given. Numerical results verify the effectiveness of theoretical analysis. What's more, we also give a conjecture about the relationship between eigenvalues and the type of periodic orbits when eigenvalues are imaginary numbers.

Keywords: Cantilever beam system with impacts; Grazing bifurcation; Discontinuous mapping; Existence of periodic orbits; Stability.

\section{Introduction}

Because of the existence of collision, vibro-impact system is strongly nonlinear and nonsmooth, and has various nonclassical bifurcations caused by grazing. Shaw ${ }^{[1]}$ considered a class of elastically constrained impact vibration systems, and found that the zero-velocity impact will cause

\footnotetext{
${ }^{*}$ Corresponding author.
}

E-mail addresses: leyuan2003@sina.com (Yuan Yue),run139315@163.com (Run Liu),jhxie2000@126.com (Jianhua Xie) 
the singularity of the Poincaré map. Whiston ${ }^{[2]}$ firstly studied the non-differentiable of Poincare mapping near the grazing point by using the singularity theory, and analyzed the reason of the singularity generated by the breaking of stable manifolds. Nordmark ${ }^{[3]}$ considered rigidly constrained impact oscillator with periodic excitation, introduced the concept of discontinuous mapping to study the singularity of the system caused by grazing bifurcations by analytic method, and found that grazing bifurcation makes a stable periodic motion into a chaotic state. In Ref.[4], Nordmark analyzed the universal limit mapping of a kind of impact oscillator when the eigenvalues are real numbers, and converted the system into the form of interval mapping. It is proved that there is no periodic orbit when the maximum eigenvalue is greater than two-thirds, and the limit mapping for any parameter is chaotic state. Dankowicz ${ }^{[5]}$ studied a class of micro-drive devices, proposed three types of co-dimension one bifurcation types, and used the discontinuous mapping method to predict the dynamic characteristics of the grazing bifurcation based on the system properties. Zhao ${ }^{[6]}$ derived the discontinuous mapping of a single-degree-of-freedom impact oscillator, and uncovered the relationship between the discontinuous mapping of the spring oscillator and its actual physical parameters. Bernard ${ }^{[7]}$ analyzed the slip bifurcations of $\mathrm{n}$-dimensional piecewise smooth dynamical systems, gave the corresponding discontinuous mapping, and derived the complete expression of the grazing-induced bifurcation normal form mapping. $\mathrm{Li}^{[8]}$ considered a two-degree-of-freedom impact oscillator with symmetrical rigid constraints, found the dynamic behavior induced by grazing bifurcation numerically. Zhang ${ }^{[9]}$ derived the discontinuous maps of a rub-impacting rotor system, used the sequence limit theory to analyze the bifurcation situation induced by grazing of the rotor system. Feng ${ }^{[10]}$ used the graph cell mapping theory to study the global dynamics of the grazinginduced crisis of the Duffing system. Luo ${ }^{[11]}$ studied a kind of two-degree-of-freedom vibration system with clearance, and revealed the influence of the grazing bifurcation on the global bifurcation. Shen ${ }^{[12-13]}$ considered an impact oscillator, suppressed the jumping phenomenon at the grazing bifurcation point based on the special dynamic characteristics of the continuous transition at the degenerated grazing bifurcation point. Simpson ${ }^{[14]}$ considered a kind of impact oscillators with random excitation, and gave the dynamic behavior induced by grazing under different random excitation. Simpson ${ }^{[15]}$ considered a class of single-degree-of-degree impact oscillators, which revealed that some truncated terms neglected in the Nordmark mapping had important effects on 
the dynamics characteristics of the real primary system. $\mathrm{Xu}^{[16]}$ studied the grazing bifurcation problem of a class of 2-DOF impact systems with clearance, and proposed a discrete time feedback method to control the stability of periodic orbits induced by the grazing bifurcation. Jiang ${ }^{[17]}$ studied the grazing bifurcation of impact oscillators under elastic and rigid constraints by using the path tracking method, and calculated the bifurcation points under different stiffness and recovery coefficients. Brzeski ${ }^{[18]}$ studied the grazing bifurcation problem of a kind of church pendulum model with clearance, and revealed a new grazing bifurcation phenomenon different from the period adding motion, that is, " impact adding" phenomenon. Yin ${ }^{[19]}$ considered the single-degree of freedom impulse oscillator, and used the time-discrete control method to control the unstable orbit after the grazing bifurcation. In Ref.[20], GPU parallel computing technology is used to study the two-parameter dynamics problem in the neighborhood of grazing bifurcation points.

There is important significance to study the dynamics of beam structures with impact. Emans ${ }^{[21]}$ first introduced the nonlinear restoring force to establish the nonlinear elastic impact model, and studied the chaotic phenomenon by numerical simulation. Wang ${ }^{[22]}$ carried out global dynamics analysis on the situation of the one-side elastic impact and bilateral impact of the system. Huangfu ${ }^{[23]}$ conducted a numerical analysis of unilateral grazing bifurcation and verified that the local discontinuous mapping is also effective when it contains nonlinear terms.

This paper focuses on the existence and stability of the periodic orbits induced by grazing in a cantilever beam system with impacts. In Sect. 2, the mathematical model and the dynamical equations are represented briefly. In Sect. 3, the discontinuous mapping method is used to established the Poincaré compound mapping, and a combination of inhomogeneous equations and inequations is obtained to determine the existence and stability of the periodic orbits induced by grazing bifurcations. In addition, in the case that the eigenvalues are imaginary numbers, a conjecture about the relationship between eigenvalues and the type of periodic orbits is given. In Sect. 4, numerical simulations verify the effectiveness of the theoretical analysis. Conclusions are given in Sect.5. 


\section{Cantilever Beam system with impacts}

The cantilever beam system with impacts is shown in Fig.1. The mass $M$ is subjected to harmonic excitation, and the cantilever beam is simplified into two plate springs neglecting of the mass. The length of plate springs is $L$. The bending stiffness is $E I$, where $E$ is elastic modulus, and I is rotational inertia. The Cartesian coordinate was established as shown in Fig.1. The cantilever beam is assumed to undergo small amplitude vibration.

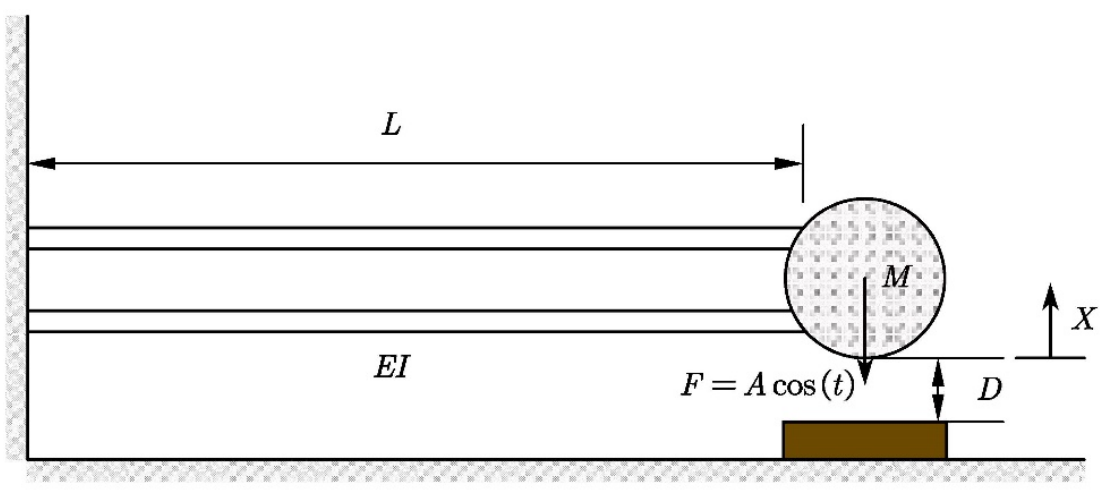

Fig.1. A cantilever beam system with one sided impacts.

When there is no impact, the differential equation of motion of the mass $M$ is:

$$
M \ddot{X}+c \dot{X}+a(X)=A \cos (t) \text {, as } X>-D \text {. }
$$

The collision is described by Newton impact model:

$$
\dot{X}_{+}=U\left(\dot{X}_{-}\right)=-r \dot{X}_{-} \text {, as } X=-D,
$$

where $r$ is Newton's coefficient of restitution of impact $(r \in(0,1))$, which $\dot{X}_{+}$and $\dot{X}_{-}$denote the velocities after and before the impact, respectively. In Eq.(1), the elastic restoring force $a(X)$ is described as a nonlinear form ${ }^{[21]}$

$$
a(X)=\frac{12 E I}{L^{3}} X+\frac{432 E I}{35 L^{5}} X^{3} .
$$

Let $q=\frac{X}{L}$, after being dimensionless, Eq. (1)can be described as a two dimensional first-order nonlinear system 


$$
\left\{\begin{array}{c}
\dot{q}=u \\
\dot{u}=-\zeta u-\alpha\left(q+\frac{36}{35} q^{3}\right)+\beta \cos (t)
\end{array}\right.
$$

where $\zeta=\frac{c}{M}, \alpha=\frac{12 E I}{M L^{3}}, \beta=\frac{A}{M L}, d=\frac{D}{L}$. When $q=d$, the mass block impacts with the constraint, and the velocity changes as: $\dot{u}_{+}=-r \dot{u}_{-}$according to Newton's impacting law.

\section{Existence and stability of the grazing-induced periodic orbits}

The impact clearance is selected as the bifurcation parameter, and the position constraints is given by $Q=d+\mu$, where $\mu$ is the small perturbation. When there is no impact, the system belongs to a smooth dynamic system. If the mass contacts with the impact surface at zero speed, grazing takes place, which may induce various nonclassical bifurcations. The periodic orbit of the system can be determined by the shooting method numerically. In order to study the stability and dynamics of the periodic orbits after grazing, a local Poincare section is introduced. Then the periodic orbit of the original system corresponds the fixed point of the Poincare map, and the dynamics of the periodic orbit is equivalent to that of the fixed point of the Poincare map. The equations of motion are smooth slightly beyond the impact surface. However, when the grazing takes place, it is non-differentiable. The existence and stability of the grazing-induced periodic orbits can be further revealed by introducing a set of inhomogeneous equations and inequations ${ }^{[24]}$.

\section{1 Establishing the Poincaré compound mapping by using the discontinuous mapping method}

Introducing the time Poincaré cross section $(\bmod T)$, the left side of Eq.(4) is a function of period $T=2 \pi$.It is assumed that when $t=t_{G}$, the grazing happens, and at this moment $a_{G}=\dot{u}_{t_{G}}>0$. Then we introduce the time Poincaré cross section to obtain a simpler impact 
mapping form $g$. Let the section time $t$ be close to $t_{G}\left(t \neq t_{G}\right)$. In the vicinity of $t_{G}$, the time $t$ can be slightly smaller or slightly larger than $t_{G}$. We use the flow of the system (4) to convert the time to $t=t_{G}$. In this way, the Poincaré section approaches to $t=t_{G}$, and then the low-speed impact must occur either before or after $t_{G}$.

State coordinates on the Poincaré section are chosen as:

$$
\boldsymbol{x}=\left[\begin{array}{l}
x_{1} \\
x_{2}
\end{array}\right]=\left[\begin{array}{c}
q-Q \\
u
\end{array}\right]
$$

When there is not impact, the Poincare mapping of system (4) is smooth at $\boldsymbol{x}=0$ and $\mu=0$, and can be described by the following function: $\boldsymbol{f}(\boldsymbol{x}, \mu): R^{2} \times R^{1} \rightarrow R^{2}$, where $\boldsymbol{f}(0,0)=0$. However, the accurate analytical form of the Poincaré mapping cannot be obtained. Although there are some methods that can be used to obtain the approximate solutions, they rely generally on the presupposition of small perturbation of system parameters, and are only suitable for weakly nonlinear systems. First, according to the shooting method, we can obtain the periodic orbit and its Jacobi matrix $\boldsymbol{A}$ :

$$
\boldsymbol{A}=\left(D_{x} \boldsymbol{f}\right)(0,0)=\left[\begin{array}{ll}
v & \gamma \\
\rho & \delta
\end{array}\right] .
$$

Next, the zero-time discontinuous mapping $\boldsymbol{g}$ is introduced to denote the process of the lowspeed impacts, then the Poincare mapping of the whole system can be expressed as $\boldsymbol{P}=\boldsymbol{g} \circ \boldsymbol{f}$, where $\boldsymbol{g}(\boldsymbol{x}, \mu): R^{2} \times R^{1} \rightarrow R^{2}$ 。In order to indicate whether the grazing bifurcation occurs nearby $\boldsymbol{x}=0$, it is necessary to introduce a smooth function $h(\boldsymbol{x}, \mu): R^{2} \times R^{1} \rightarrow R^{1}$. The range of the function $h(\boldsymbol{x})$ is a set containing 0 , which is consistent with whether impact occur. $h(\boldsymbol{x})$ takes a negative value when a low-speed impact occurs, and a positive value when no impact occurs. Functions $h(\boldsymbol{x}, \mu)$ must satisfy $h(0,0)=0$. Let $\boldsymbol{C}=D_{x} h(0,0)$, and choosing $\boldsymbol{C}=\left[\begin{array}{ll}1 & 0\end{array}\right]$, we have $h(\boldsymbol{x})=x_{1}+h . o . t$. However, if $\boldsymbol{x}$ is close to 0 , because $a_{G}>0$, there will be a local minimum of $h(\boldsymbol{x})$. And if $\boldsymbol{h}(\boldsymbol{x})<0$, there are two intersection points between the system orbit 
and the impact surface.

It is guaranteed by the implicit function theorem that if $\operatorname{det}(\boldsymbol{I}-\boldsymbol{A}) \neq 0$, there exists an isolated fixed point $\tilde{x}(\mu)$ for small parameters $\mu$, where $\tilde{x}(0)=0$. The fixed point satisfies:

$$
\tilde{x}(\mu)=\boldsymbol{f}(\tilde{x}(\mu), \mu)
$$

Assuming that when the parameter $\mu$ changes, the equilibrium point crosses through $h=0$, then

$$
h(\tilde{x}(\mu), \mu))=e \mu+O\left(\mu^{2}\right)
$$

where

$$
e=D_{\mu}(h(\tilde{x}(\mu), \mu))(0)=\boldsymbol{C}(\boldsymbol{I}-\boldsymbol{A})^{-1}\left(D_{\mu} \boldsymbol{f}\right)(0,0)+\left(D_{\mu} h\right)(0,0) .
$$

If $e \mu$ is small and positive, $\tilde{x}(\mu)$ is a non-impact equilibrium point for the whole composite mapping $\boldsymbol{P}$. That is, if $h$ is positive, $\boldsymbol{g}$ is an identity mapping. In the case that $h$ is negative and the impact surface is not considered, the local zero-time discontinuous mapping near the grazing point at time $t=t_{G}$ is shown in Fig. 2. In this figure the solid line represents the actual flow of the system, and the dashed line represents the extended flow. To construct the discontinuity mapping, we need to know the fate of two different types of trajectory with initial conditions close to $x^{*}$. In the case that some trajectories do not cross $\Sigma$ locally(i.e., $h\left(x^{*}\right) \geq 0$ ), we have $\boldsymbol{g}\left(x^{*}\right)=x^{*}$.In contrast, the discontinuity mapping will be non-trivial for the trajectory that passes through $\Sigma$, and the trajectory hits $\Sigma$ at the point $x_{1}{ }^{*}$ at time $t_{G}-\delta$.And then it is mapped to the point $x_{2}{ }^{*}$ by the map $U\left(x_{1}^{*}\right)$ (see Eq. (2)).By extending the smooth flow field $\boldsymbol{F}(x)$ defined in the region $h(x) \geq 0$ ( above $\Sigma$ ) to the region $h(x)<0$ (below $\Sigma$ ), we may continue the trajectory forward from $x_{1}{ }^{*}$ under the action of the flow map $\phi$,or equally backward form $x_{2}{ }^{*}$. The same trajectory starting from $x_{2}{ }^{*}$ can also be continued backward under the action of $\phi$ for a time $\delta$ so that it passes through the point $x_{3}{ }^{*}$ at the time $t_{G}$. So if $h\left(x^{*}\right)<0$, the mapping process can be expressed as $x_{0}{ }^{*} \rightarrow x_{1}^{*} \rightarrow x_{2}{ }^{*} \rightarrow x_{3}{ }^{*}$. 


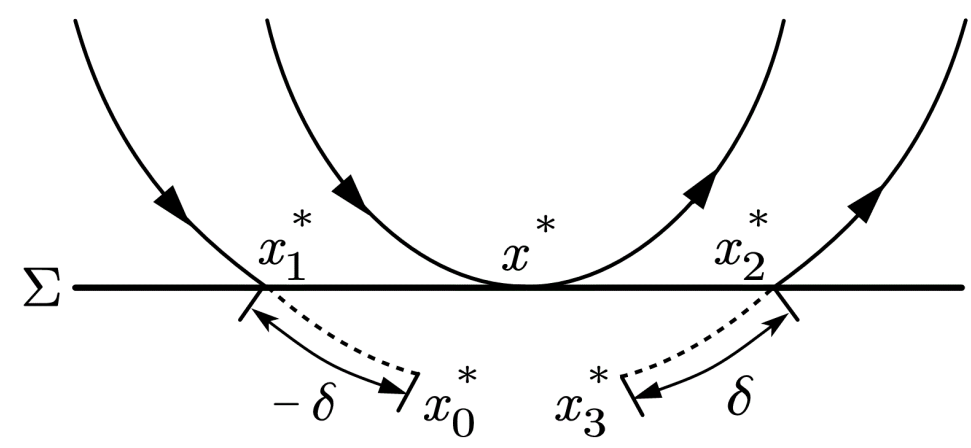

Fig. 2. Schematic diagram of zero time discontinuous mapping $g$.

The local discontinuous mapping expression near the grazing bifurcation point can be written as



The following derivation is given for zero-time discontinuous mapping.

First, we consider process $x_{0}{ }^{*} \rightarrow x_{1}{ }^{*}$ which takes time $\delta$. The first order Taylor expansion of the flow function $\phi$ with respect to time can be expressed as

$$
x_{1}^{*}=\boldsymbol{\phi}\left(x_{0}{ }^{*},-\delta\right)=x_{0}^{*}-\boldsymbol{F}\left(x_{0}^{*}\right) \delta+o(\delta)
$$

where $\boldsymbol{F}\left(x^{*}\right)$ represents the vector field at $x^{*}$. Since $x_{0}{ }^{*}$ is in a small neighborhood of $x^{*}$, we have $\boldsymbol{F}\left(x_{0}^{*}\right)=\boldsymbol{F}\left(x^{*}\right)$. The Taylor expansion of the expression $\boldsymbol{h}\left(x_{1}{ }^{*}, \mu\right)$ at $\boldsymbol{x}=x^{*}$ and $\delta=0 \quad$ can be expressed as

$$
\begin{aligned}
\boldsymbol{h}\left(x_{1}^{*}, \mu\right) & =\boldsymbol{h}\left(\boldsymbol{\phi}\left(x_{0}{ }^{*},-\delta\right), \mu\right) \\
& =\boldsymbol{h}\left(x_{0}^{*}, \mu\right)-D_{t} \boldsymbol{h}\left(x_{0}{ }^{*}, \mu\right) \delta+\frac{1}{2} a\left(x_{0}{ }^{*}, \mu\right) \delta^{2}+o\left(\delta^{2}\right),
\end{aligned}
$$

where $D_{t} \boldsymbol{h}\left(x_{0}{ }^{*}, \mu\right)=0$, and $a\left(x_{0}{ }^{*}, \mu\right)=a_{G}$. Because $x_{1}{ }^{*}$ is on the impact surface $\Sigma$, where $\boldsymbol{h}\left(x_{1}^{*}, \mu\right)=0$ holds, we have

$$
\delta=\sqrt{\frac{\boldsymbol{h}\left(x_{0}{ }^{*}, \mu\right)}{a_{G}}}+\text { h.o.t. }
$$

Second, considering the process $x_{1}{ }^{*} \rightarrow x_{2}{ }^{*}$, we have 


$$
x_{2}^{*}=\boldsymbol{U}\left(x_{1}^{*}\right)=\boldsymbol{U}\left(\phi\left(x_{0}{ }^{*},-\delta\right)\right)=x^{*}+\boldsymbol{U}_{X}\left(\phi\left(x_{0}^{*},-\delta\right)-x^{*}\right)+\text { h.o.t },
$$

where $\boldsymbol{U}_{X}=\left[\begin{array}{cc}1 & 0 \\ 0 & -r\end{array}\right]$.

Third, considering the process $x_{2}{ }^{*} \rightarrow x_{3}{ }^{*}$, we have

$$
x_{3}^{*}=\phi\left(x_{2}^{*}, \delta\right)=x_{2}^{*}+\boldsymbol{F}\left(x^{*}\right) \delta+\text { h.o.t. }
$$

Substituting Eqs.(13) and (14) into Eq.(15),we obtain

$$
x_{3}^{*}=x^{*}+\left(\boldsymbol{F}\left(x^{*}\right)-\boldsymbol{U}_{X} \boldsymbol{F}\left(x^{*}\right)\right) \sqrt{\frac{-2 h\left(x_{0}{ }^{*}, \mu\right)}{a_{G}}}+\text { h.o.t },
$$

where $\boldsymbol{F}\left(x^{*}\right)=\left[\begin{array}{ll}0 & a_{G}\end{array}\right]^{T}$. Therefore, the expression of the zero-time discontinuous mapping of the grazing point can be written as

$$
\boldsymbol{g}(\boldsymbol{x}, \mu)= \begin{cases}\boldsymbol{x}, & \text { as } h(\boldsymbol{x}, \mu) \geq 0, \\
\boldsymbol{x}+\left[\begin{array}{l}
0 \\
1
\end{array}\right](1+r) \sqrt{2 a_{G}} \sqrt{-h(\boldsymbol{x}, \mu)}+\text { h.o.t, } & \text { as } h(\boldsymbol{x}, \mu)<0,\end{cases}
$$

let $y=\sqrt{-h(\boldsymbol{x}, \mu)}, \boldsymbol{B}=\left[\begin{array}{l}0 \\ 1\end{array}\right] \sqrt{2 a_{G}}(1+r)$, Eq.(17) can be rewritten as

$$
\boldsymbol{g}(\boldsymbol{x}, \mu)= \begin{cases}\boldsymbol{x}, & \text { as } h(\boldsymbol{x}, \mu) \geq 0 \\ \boldsymbol{x}+\boldsymbol{B} y+\text { h.o.t, } & \text { as } h(\boldsymbol{x}, \mu)<0\end{cases}
$$

\section{2 Determining the periodic orbit}

The period orbit of the original system is equivalent to the period fixed point of the composite mapping. The fixed point of $n$-period should satisfy: $\bar{x}=(\boldsymbol{g} \circ \boldsymbol{f})^{n}(\bar{x}, \mu)$. However, due to the existence of different expressions of $\boldsymbol{g}$ and singularities at $h=0$, the solving process is very difficult. Therefore, first it is necessary to distinguish between $h>0$ and $h<0$, where $h<0$ represents a low-speed impact, and $h>0$ represents no impact.

Non-impact periodic orbits meet

$$
\bar{x}-f^{n}(\bar{x}, \mu)=0
$$

where the restriction is 


$$
h\left(f^{k}(\bar{x}, \mu), \mu\right)>0, \quad 1 \leqslant k \leqslant n,
$$

and the linear stability is

$$
\left(D_{x}(\boldsymbol{g} \circ \boldsymbol{f})^{n}\right)(\bar{x}, \mu)=\left(D_{x} \boldsymbol{f}^{n}\right)(\bar{x}, \mu) .
$$

In order to simplify the analysis process, we use the term $1 / n$-period motion to represent one low-speed impact occurs in the $n$-period orbit. That is, $n-1$ non-impact iterations are followed by one impact iteration. In this way, the square root singularity of Eq.(17) can be ignored. Then we have

$$
\left\{\begin{array}{c}
\bar{x}-\boldsymbol{B} y-\boldsymbol{f}^{n}(\bar{x}, \mu)=0, \\
h\left(\boldsymbol{f}^{n}(\bar{x}, \mu), \mu\right)+y^{2}=0
\end{array}\right.
$$

where

$$
h\left(f^{k}(\bar{x}, \mu), \mu\right)>0, \quad 1 \leqslant k \leqslant n-1,
$$

and we can solve $y$ as follows:

$$
y=\sqrt{-h\left(f^{n}(\bar{x}, \mu), \mu\right)}>0 .
$$

Now we linearize this Poincaré map to determine the stability of the periodic orbits. The linearizing expression is:

$$
\left(D_{x}(\boldsymbol{g} \circ \boldsymbol{f})^{n}\right)(\bar{x})=\left[\boldsymbol{I}-(\boldsymbol{B} / y)\left(D_{x} h\right)\left(\boldsymbol{f}^{n}(\bar{x}, \mu), \mu\right) / 2\right]\left(D_{x} \boldsymbol{f}^{n}\right)(\bar{x}, \mu) .
$$

When $\mu$ changes, the equilibrium point crosses the cross section. That is, $y=O(\mu)$ holds when $\mu \rightarrow 0$. It can be concluded that the first term of Equation (25) is:

$$
\left(D_{x}(\boldsymbol{g} \circ \boldsymbol{f})^{n}\right)(\overline{\boldsymbol{x}})=-\boldsymbol{B} \boldsymbol{C} \boldsymbol{A}^{n} /(2 y)+\text { h.o.t.. }
$$

When $\mu \rightarrow 0$, at the same time $y=O(\mu)$,Eq.(26) tends to infinity. Hence, if $\operatorname{det}(\boldsymbol{I}-\boldsymbol{A}) \neq 0$ hold, the orbit will lose its stable state. What's more, when $\mu \rightarrow 0$, from the perspective of stability, the low-period orbit will lose its stability and translates to the high-period orbit, which will be discussed further below. 


\section{3 Existence of periodic orbits induced by grazing}

In order to make the representation of (22)-(24) clearer, new coordinates $\boldsymbol{x}=\tilde{x}(\mu)+e \mu \boldsymbol{Z}$, $y=e \mu Y$ are introduced. Since $h(\boldsymbol{x})$ is a function of $q$ and $Q$, By formula (9), we have $e=-1$. According to Eq.(7), we obtain

$$
\begin{aligned}
\boldsymbol{f}^{k}(\boldsymbol{x}, \mu) & =\boldsymbol{f}^{k}(\tilde{x}(\mu)-\mu \boldsymbol{Z}, \mu) \\
& =\boldsymbol{f}^{k}(\tilde{x}(\mu), \mu)-\boldsymbol{\mu} \boldsymbol{A}^{k} \boldsymbol{Z}+O\left(\mu^{2}\right)=\tilde{x}(\mu)-\mu \boldsymbol{A}^{k} \boldsymbol{Z}+O\left(\mu^{2}\right) .
\end{aligned}
$$

According Eq.(8), we have

$$
\begin{aligned}
h\left(\boldsymbol{f}^{k}(\boldsymbol{x}, \mu), \mu\right) & =h\left(\tilde{x}(\mu)-\mu \boldsymbol{A}^{k} \boldsymbol{Z}+O\left(\mu^{2}\right), \mu\right) \\
& =h(\tilde{x}(\mu), \mu)-\mu \boldsymbol{C A}^{k} \boldsymbol{Z}+O\left(\mu^{2}\right)=-\mu-\mu \boldsymbol{C A}^{k} \boldsymbol{Z}+O\left(\mu^{2}\right) .
\end{aligned}
$$

Then Eq.(22) can be changed as

$$
\left\{\begin{array}{c}
\mu\left(\boldsymbol{Z}-\boldsymbol{B} \boldsymbol{Y}-\boldsymbol{A}^{n} \boldsymbol{Z}+O(\mu)\right)=0, \\
\mu\left(1+\boldsymbol{C A}^{n} \boldsymbol{Z}+O(\mu)\right)=0
\end{array}\right.
$$

that is

$$
\left[\begin{array}{cc}
\boldsymbol{I}-\boldsymbol{A}^{n} & -\boldsymbol{B} \\
\boldsymbol{C A}^{n} & 0
\end{array}\right]\left[\begin{array}{c}
\boldsymbol{Z} \\
Y
\end{array}\right]=\left[\begin{array}{c}
0 \\
-1
\end{array}\right]+O(\mu)
$$

where the restrictions are

$$
\operatorname{sign}(\mu)\left(\boldsymbol{C A}^{k} \boldsymbol{Z}+1\right)+O(\mu)<0, \quad 1 \leqslant k \leqslant n-1,
$$

and

$$
\operatorname{sign}(\mu) Y<0 \text {. }
$$

When there is a grazing impact of the original trajectory(i.e. $\mu \rightarrow 0+$ ), Eqs.(30)-(32) can be changed as

$$
\begin{aligned}
& {\left[\begin{array}{cc}
\boldsymbol{I}-\boldsymbol{A}^{n} & -\boldsymbol{B} \\
\boldsymbol{C A}^{n} & 0
\end{array}\right]\left[\begin{array}{l}
\boldsymbol{Z} \\
Y
\end{array}\right]=\left[\begin{array}{c}
0 \\
-1
\end{array}\right] .} \\
& \boldsymbol{C A}^{k} \boldsymbol{Z}+1<0, \quad 1 \leqslant k \leqslant n-1 . \\
& Y<0 \text {. }
\end{aligned}
$$

Eqs.(33)-(35) determine the existence of periodic solutions after grazing impact. For 
simplicity ,Eq.(33) is rewritten as $\boldsymbol{D} \mathbb{Z}(\mu)=\left[\begin{array}{ll}0 & -1\end{array}\right]^{T}$. The following lemma gives the sufficiency conditions of the existence of $1 / n$-period motion induced by grazing impact.

Lemma 1.(Existence of $1 / n$-period motions induced by grazing impact.)Assuming that $\operatorname{det}(\boldsymbol{I}-\boldsymbol{A}) \neq 0, \operatorname{det}(\boldsymbol{D}) \neq 0$, and let $\mathbb{Z}(0)$ be the solution of Eq.(33). If the solution $\mathbb{Z}(\mu)(\mu \rightarrow 0+)$ satisfies Eqs.(34)-(35), then there is a unique $1 / n$-period motion induced by grazing, which approaches $\boldsymbol{x}=0$ as $\mu \rightarrow 0+$. Conversely, if $\mathbb{Z}(\mu)(\mu \rightarrow 0+)$ does not satisfy either of Eqs.(34) and (35), then there is not a $1 / n$-periodic motion.

Lemma 1 provides a sufficient condition for the existence of $1 / n$-period motions induced by grazing, but there are still some other cases which are not included. For example, if there are some characteristic multipliers of the non-impact fixed point locating on the unit circle, then it will be necessary to discuss the smooth bifurcation of the original system and then make a further discussion. In the case of $\operatorname{det}(\boldsymbol{D})=0$, the implicit function theorem does not hold, there may be multiple solutions which be stable or not.

Although we obtain a combination of non-homogeneous equations and inequalities to determine the existence of the $1 / n$-period motions induced by grazing, Eqs.(33)-(35) are still somewhat difficult to be solve. Hence, other simple equations about the eigenvalues are obtained through the following transformations. It should be mentioned that, when $\gamma=0$ in the Jacobi matrix $\boldsymbol{A}, \boldsymbol{A}^{n}$ should be upper triangular matrices, and $\boldsymbol{A}^{n} \boldsymbol{B}$ is parallel with $\boldsymbol{B}$. Subsequently the null space of $\boldsymbol{D}$ can be constructed. So if $\operatorname{det}(\boldsymbol{D}) \neq 0($ i.e. $\gamma \neq 0)$, the matrices $\boldsymbol{A}, \boldsymbol{B}$ and $\boldsymbol{C}$ can be transformed into the following form ${ }^{[3]}: \quad \boldsymbol{A}=\left[\begin{array}{cc}\lambda_{1}+\lambda_{2} & 1 \\ -\lambda_{1} \lambda_{2} & 0\end{array}\right], \quad \boldsymbol{B}=\left[\begin{array}{l}0 \\ 1\end{array}\right] \sqrt{2 a_{G}}(1+r) \gamma$ and $\boldsymbol{C}=\left[\begin{array}{ll}1 & 0\end{array}\right]$. Here $\lambda_{1}$ and $\lambda_{2}$ are the eigenvalues of $\boldsymbol{A}, \lambda_{1}+\lambda_{2}=v+\delta$ is the trace of $\boldsymbol{A}$, and $\lambda_{1} \lambda_{2}=v \delta-\rho \gamma$ is the determinant of $\boldsymbol{A}$. It should be noted that if $\gamma$ is small, this transformation is singular and large deformation is introduced, which is not the content of this article. First we eliminate $Y$ in Eq.(33), and rewrite Eqs.(33)-(35) as 


$$
\begin{gathered}
{\left[\begin{array}{cc}
{\left[\begin{array}{cc}
1 & 0
\end{array}\right]\left(I-\boldsymbol{A}^{n}\right)} \\
{\left[\begin{array}{ll}
1 & 0
\end{array}\right]}
\end{array}\right] \boldsymbol{Z}=\left[\begin{array}{c}
0 \\
-1
\end{array}\right],} \\
b_{n, k}<0, \quad 1 \leqslant k \leqslant n-1, \\
\operatorname{sign}(\gamma) c_{n}<0 .
\end{gathered}
$$

where,

$$
\begin{aligned}
& b_{n, k}=\left[\begin{array}{ll}
1 & 0
\end{array}\right] \boldsymbol{A}^{k} \boldsymbol{Z}+1, \\
& c_{n}=\left[\begin{array}{ll}
0 & 1
\end{array}\right]\left(\boldsymbol{I}-\boldsymbol{A}^{n}\right) \boldsymbol{Z},
\end{aligned}
$$

we have

$$
\boldsymbol{A}^{n}=\frac{1}{\lambda_{1}-\lambda_{2}}\left[\begin{array}{cc}
\lambda_{1}^{n+1}-\lambda_{2}^{n+1} & \lambda_{1}^{n}-\lambda_{2}^{n} \\
-\lambda_{1} \lambda_{2}\left(\lambda_{1}^{n}-\lambda_{2}^{n}\right) & -\lambda_{1} \lambda_{2}\left(\lambda_{1}^{n-1}-\lambda_{2}^{n-1}\right)
\end{array}\right],
$$

where $\lambda_{1} \neq \lambda_{2}$ holds. Solving Eq.(36), we have

$$
\boldsymbol{Z}=\left[\begin{array}{c}
-1 \\
Z_{2}
\end{array}\right]
$$

where

$$
\begin{gathered}
Z_{2}=\frac{\left(\lambda_{1}^{n+1}-\lambda_{2}^{n+1}\right)-\left(\lambda_{1}-\lambda_{2}\right)}{\lambda_{1}^{n}-\lambda_{2}^{n}}, \\
b_{n, k}=\frac{-\left(\lambda_{1} \lambda_{2}\right)^{k}\left(\lambda_{1}^{n-k}-\lambda_{2}^{n-k}\right)-\left(\lambda_{1}^{k}-\lambda_{2}^{k}\right)+\left(\lambda_{1}^{n}-\lambda_{2}^{n}\right)}{\lambda_{1}^{n}-\lambda_{2}^{n}}, \\
c_{n}=\frac{-\left(\lambda_{1}-\lambda_{2}\right)\left(\lambda_{1}^{n}-1\right)\left(\lambda_{2}^{n}-1\right)}{\lambda_{1}^{n}-\lambda_{2}^{n}} .
\end{gathered}
$$

The following Lemma give the relationship between the existence of periodic orbits and the eigenvalues.

Lemma 2.(The relationship between the existence of periodic motion and the eigenvalues)Assuming that the non-impact fixed point is a stable hyperbolic one, $\lambda_{1} \neq \lambda_{2}$ and $\operatorname{det}(\boldsymbol{D}) \neq 0$. If both Eqs.(37) and (38) hold, then there is a unique $1 / n$-period motion induced by grazing. If their don't satisfy either of Eqs.(37) and (38), there is no $1 / n$-period motion.

Returning to the question of stablity, the maximum modulus of Eq.(26) can be given as 


$$
\begin{aligned}
\Lambda & =-\boldsymbol{B C}^{n} /(2 y)+\text { h.o.t } \\
& =-\frac{a_{G}(1+r)^{2} \gamma^{2}}{\mu}\left(\frac{\lambda_{1}^{n}-\lambda_{2}^{n}}{\lambda_{1}-\lambda_{2}}\right)^{2} \frac{1}{\left(\lambda_{1}^{n}-1\right)\left(\lambda_{2}^{n}-1\right)}+\text { h.o.t.. }
\end{aligned}
$$

If $\mu \rightarrow 0+$, the value of $\Lambda$ tends to infinity, indicating that the original periodic orbit will lose the stability. If there is still a steady motion after grazing, the original periodic orbit will bifurcate into same high-period one or chaotic one, which is verified by the numerical analysis in section 4.

Through the above transformation, a combination of equations and inequalities are obtained to determine the dynamics near grazing point. In this way, the sufficient conditions for the existence of periodic solutions and the stability criteria of these orbits can be obtained.

\section{4 Determing the type of the periodic motion induced by grazing by the eigenvalues}

In this section, we use lemma 2 to discuss in detail of the different eigenvalue cases.

Case I. Eigenvalues are reals.

In this case, supposing $0<\lambda_{2}<\lambda_{1}<1$, according to Eq.(45), no matter how $n$ changes, $c_{n}$ must be negative. By Eq.(44), we get

$$
b_{n, k}=\frac{\left(\lambda_{1}-1\right)\left(1-\lambda_{2}\right)\left(\lambda_{1}-\lambda_{2}\right) \sum_{i=k}^{n-1} \sum_{j=0}^{k-1} \sum_{m=j}^{i-1} \lambda_{1}^{m} \lambda_{2}^{i+j-m-1}}{\left(\lambda_{1}-\lambda_{2}\right) \sum_{i=0}^{n-1} \lambda_{1}^{i} \lambda_{2}^{n-i-1}},
$$

then $b_{n, k}$ also takes a negative value. Based on Eqs.(36)-(38) and Lemma 2, the following theorem which describe the relationship between eigenvalues and the type of periodic motions induced by grazing can be obtained.

Theorem 1.(Determing the type of the periodic motion induced by grazing by the real eigenvalues) Assuming the non-impact fixed point is a stable hyperbolic one, $\lambda_{1} \neq \lambda_{2}$ and $\operatorname{det}(\boldsymbol{D}) \neq 0$. If both $\lambda_{1}$ and $\lambda_{2}$ are reals, then the sufficient condition for the existence of a 
unique $1 / n(n \geq 1)$ periodic motion induced by grazing is $\operatorname{sign}(\gamma)>0$.

Proof. If eigenvalues $\lambda_{i}(i=1,2)$ are reals and supposing $0<\lambda_{2}<\lambda_{1}<1$, then we have $c_{n}<0$ and $b_{n, k}<0$ based on Eqs.(45) and (47). Then the sufficient condition for the existence of a unique $1 / n(n \geq 1)$ periodic motion induced by grazing is $\operatorname{sign}(\gamma)>0$ because both Eqs.(37) and (38) must hold according to lemma 2 .

Case II. Eigenvalues are a pair of conjugate complexes

In this case, the situation will be very complicated. After grazing bifurcation occurs, the condition for the existence of a $1 / 1$ periodic motion is $\operatorname{sign}(\gamma)>0$.According to Eq.(44), it can be concluded that the sufficient conditions for the existence of $1 / 2$ periodic motion are $\lambda_{1}+\lambda_{2}>0$ and $\operatorname{sign}(\gamma)>0$. However, in the case of $n \geq 3$, the signs of $c_{n}$ and $b_{n, k}$ are difficult to determine. The eigenvalue are written as $\lambda_{1,2}=\kappa e^{ \pm i \theta}(0<\theta<\pi)$, where

$$
\kappa=\sqrt{\xi^{2}+\eta^{2}}
$$

where $\xi$ is the real part of $\lambda_{1,2}$ and $\eta$ is the imaginary part of $\lambda_{1,2}$.

Then Eqs.(43)-(44) can be rewritten as

$$
\operatorname{sign}\left(b_{n, k}\right)=\operatorname{sign}\left(\frac{-\kappa^{n} \sin ((n-k) \theta)+\kappa^{n-k} \sin (n \theta)-\sin (k \theta)}{\sin (n \theta)}\right)
$$

and

$$
\operatorname{sign}\left(c_{n}\right)=-\operatorname{sign}(\sin (n \theta))
$$

let

$$
s=\theta / 2 \pi
$$

Ref.[24] has proved that, in the case of $\kappa=1$, there is a unique periodic $1 / n$-period motion if $s \in(0,1 / 2 n)$. Here, we focus on the cases of $\kappa<1$. After a large number of numerical simulations (see section 4), we have the following conjecture which uncovers the relationship between conjugate the complex eigenvalues and the type of the periodic motions induced by grazing. 
Conjecture 1(Determing the type of the periodic motion induced by grazing by the conjugate eigenvalues in the case of $\kappa<1$ ) Assuming the non-impact fixed point is a stable hyperbolic one and $\operatorname{det}(\boldsymbol{D}) \neq 0$. In the case of a pair of conjugate complex eigenvalues, then there is a unique $1 / n$-period motion induced by grazing if $s \in(1 / 2 n, 1 / 2(n-1))$.

\section{Numerical simulation}

In this section, the clearance $\mu$ is chosen as the bifurcation parameter, the coefficient of impacting restitution is fixed as $r=0.65$.

\section{1 The case of real eigenvalues}

In this section, taking the system parameters: $\alpha=0.7$ and $\beta=0.1$. For different value $\zeta$, the corresponding real eigenvalues $\lambda_{1}$ and $\lambda_{2}$ are obtained by shooting method at first, at the same time the value of $\gamma$ is computed. The results of computation are listed as table 1 . The periodic orbits induced by grazing corresponded to different value of $\zeta$ are shown as Fig.3. In the figures, $x_{1}$ represents the displacement of the cantilever beam. Here we can see that the numerical results are consistent with Theorem 1. We discuss the five cases as follows in detail.

Table 1 Eigenvalues of period orbits and $\gamma$-values

\begin{tabular}{ccccc}
\hline Fig. Name & $\zeta$ & $\lambda_{1}$ & $\lambda_{2}$ & $\gamma$ \\
\hline Fig. 3(a) & 1.7 & 0.0113614 & 0.0020216 & $\gamma=0.0339363>0$ \\
Fig. 3(b) & 2.0 & 0.0574449 & 0.0000607 & $\gamma=0.0525654>0$ \\
Fig. 3(c) & 2.5 & 0.1320600 & 0.0000012 & $\gamma=0.0711368>0$ \\
Fig. 3(d) & 3.5 & 0.2618876 & $1.2 \times 10^{-9}$ & $\gamma=0.0851969>0$ \\
Fig. 3(e) & 4.5 & 0.3625733 & $1.5 \times 10^{-12}$ & $\gamma=0.0867967>0$ \\
\hline
\end{tabular}

(I) $\zeta=1.7$.According to the stability criterion Eq.(46), there is $\Lambda_{2}=-0.1627828$ as $\mu=2 \times 10^{-7}$, indicating that the $1 / 2$ periodic motion in Fig.3(a) is stable. Here $\Lambda_{i}$ denotes the value of Eq.(46) as $n=i$. If $\mu$ is decreased to $\mu=3 \times 10^{-8}$, because $\left|\Lambda_{2}\right|=1.9739004>1$ and $\Lambda_{3}=-2.215 \times 10^{-4}$, the $1 / 2$ periodic motion will lose the stability, and bifurcates into a $1 / 3$ 
periodic motion, as shown in Fig.3(a).

(II) $\zeta=2.0$. Since the value of $\Lambda_{3}$ is $\Lambda_{3}=-0.0406154$ as $\mu=2 \times 10^{-7}$, then the $1 / 3$ periodic motion in Fig. 3(b) is stable. If $\mu$ is decreased to $\mu=2 \times 10^{-9}$, because $\left|\Lambda_{3}\right|=2.0307688>1$ and $\Lambda_{4}=-6.700 \times 10^{-4}$, the $1 / 3$ periodic motion will become unstable, and bifurcates into a 1/4 periodic motion, as shown in Fig. 3(b).

(III) $\zeta=2.5$. The value of $\Lambda_{4}$ is computed as $\Lambda_{4}=-0.0967943$ as $\mu=3 \times 10^{-8}$, hence we conclude that the $1 / 4$ periodic motion in Fig. 3(c) is stable. If $\mu$ is decreased to $\mu=2 \times 10^{-9}$, because $\left|\Lambda_{4}\right|=1.4519146>1$ and $\Lambda_{5}=-0.2531449$, the $1 / 4$ periodic motion will lose the stability, and bifurcates into a 1/5 periodic motion, as shown in Fig. 3(c).

(IV) $\zeta=3.5$. We have $\Lambda_{5}=-0.0140201$ as $\mu=4 \times 10^{-8}$,which means that the $1 / 5$ periodic motion in Fig. 3(d) is stable. If $\mu$ is decreased to $\mu=5 \times 10^{-10}$, because $\left|\Lambda_{5}\right|=1.1216107>1$ and $\Lambda_{6}=-0.0768558$, the $1 / 5$ periodic motion will lose the stability, and bifurcates into a 1/6 periodic motion, as shown in Fig.3(d).

(V) $\zeta=4.5$. There is $\Lambda_{6}=-0.0161048$ as $\mu=5 \times 10^{-8}$, which means that the $1 / 6$ periodic motion in Fig. 3(e) is stable. If $\mu$ is decreased to $\mu=3 \times 10^{-10}$, because $\left|\Lambda_{6}\right|=2.6841356>1$ and $\Lambda_{6}=-0.3523434$, the $1 / 6$ periodic motion become unstable, and bifurcates into a 1/7 periodic motion, as shown in Fig.3(e).

It should be mentioned that the stability criterion Eq.(46) only takes the leading term. Then there is an error caused by the omitted high-order term. In the case that the absolute value of the maximum eigenvalue is small, the stability criterion agrees well with the numerical simulation results, as shown in Fig. 3(a)-(c). But in the case that the absolute value of the maximum eigenvalue is larger, there may be a larger error between theoretical analysis and numerical simulation (see Fig. 3(d)-(f)). However, Eq.(46) can explain qualitatively the phenomenon of period-adding sequence very well. 


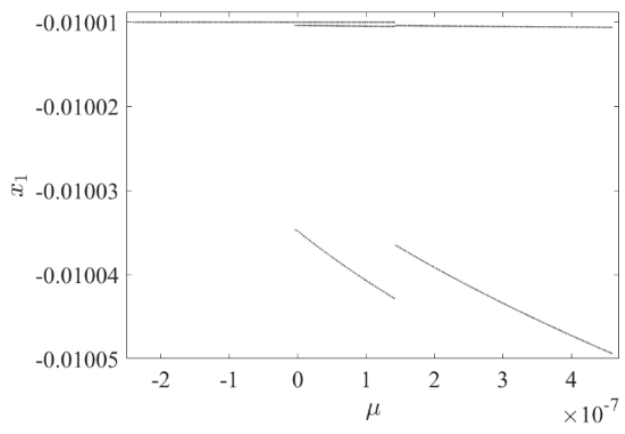

(a)

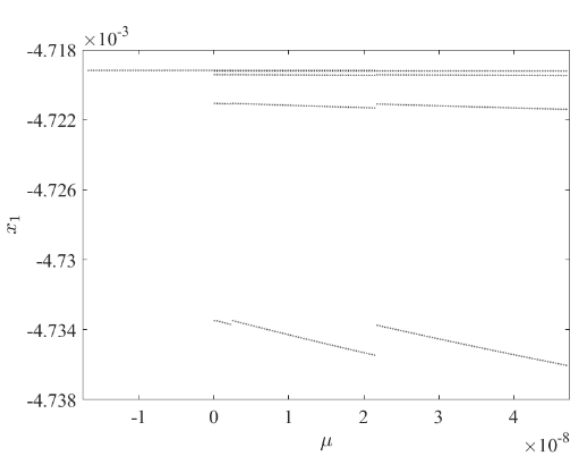

(c)

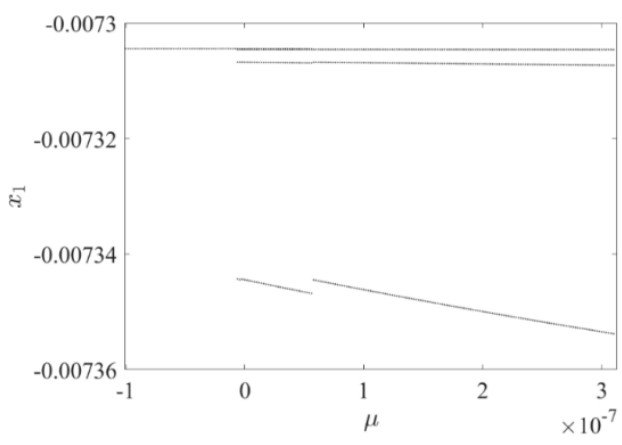

(b)

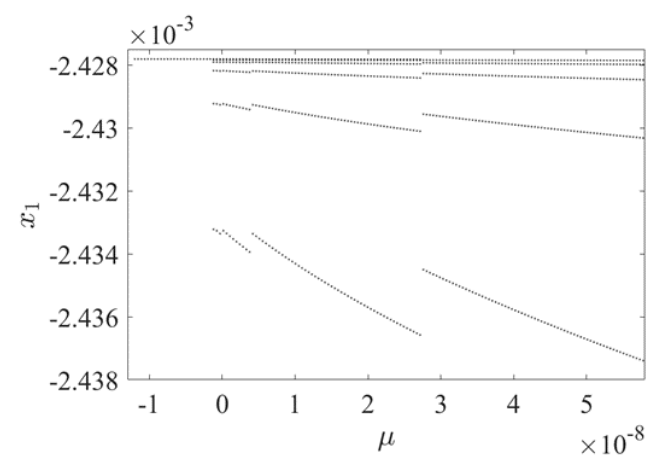

(d)

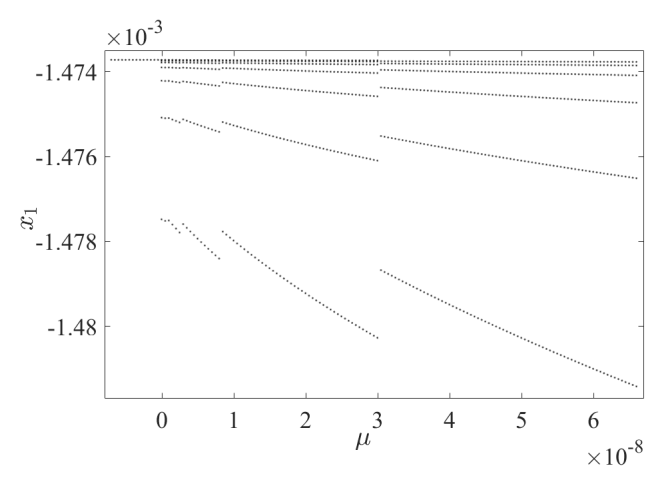

(e)

Fig. 3 The periodic orbit induced by grazing bifurcation in the case of real eigenvalues. (a) $\zeta=1.7$ (b) $\zeta=2.0 \quad$ (c) $\zeta=2.5 \quad$ (d) $\zeta=3.5 \quad$ (e) $\zeta=4.5$.

In conclusions, when eigenvalues are real numbers, the existence of periodic solutions is determined by Theorem 1 . Moreover, as $\mu \rightarrow 0+$, a period-adding bifurcation induced by grazing will occur, and Eq.(46) illustrates this process. In addition, the $n$ of $1 / n$ periodic motion induced by grazing goes up as the increase of the largest eigenvalue.

Ref.[25] summarized and classified the dynamics induced by grazing bifurcation. If the eigenvalue is a real number and $\lambda_{1}>2 / 3$, it is believed that there is generally no periodic orbit 
after grazing, but a chaotic attractor directly appears. However, here we find that the dynamics after grazing bifurcation may be more complicated. Specifically, as $\mu$ tends to $0+$, chaotic bands may alternates with periodic bands, till that the period-adding cascade appears when the parameter $\mu$ pass through some critical values. For example, taking the system parameter combination: $\zeta=3.5$, $\alpha=0.2$ and $\beta=0.2$, we can obtain $\lambda_{1}=0.6928935>2 / 3$ and $\gamma=0.2047802>0$. According to Theorem 1 , there is a $1 / n(n \geq 1)$ periodic motion induced by grazing. The bifurcation diagram is shown in Fig. 4. It is shown that when $\mu$ decrease gradually, the dynamics changes from chaotic motion to the period adding motion with chaotic bands between adjacent periodic motions. Subsequently, the chaotic bands will disappear and there is only the period-adding cascade when $\mu \rightarrow 0+$



Fig.4 The period-adding cascade induced by grazing bifurcation: $\zeta=3.5, \alpha=0.2, \beta=0.2$.

\section{2 The case of conjugate complex eigenvalues}

In the case of conjugate complex eigenvalues, we will select different parameter combinations to verify conjecture 1 . Taking the system parameter $\alpha=0.7$ and $\beta=0.1$. For different value $\zeta$, the two corresponding complex eigenvalues are obtained by shooting method, and the value of $\mathrm{s}$ is 
calculated by Eq.(51). Subsequently, we obtain the corresponding interval $(1 / 2 n, 1 / 2(n-1))$, and determine the specific $1 / n$ periodic motion induced by grazing bifurcation according to Conjecture 1. The Eigenvalues, values of $s$ and the corresponded type of periodic orbits are listed as table 2. The periodic orbits listed in table 2 are shown in Fig. 5.

(I) $\zeta=1.257$. The value of $s$ is computed as $s=0.0598100$ by Eq.(51). According to Conjecture $1, s \in(1 / 18,1 / 16)$, indicating that there is a $1 / 9$ periodic motion induced by grazing, as shown in Fig.5(a). (II) $\zeta=0.123$. The value of $s$ is $s=0.0676783$, where $s \in(1 / 16,1 / 14)$, indicating that a $1 / 8$ periodic motion induced by grazing, as shown in Fig.5(b).

(III) $\zeta=0.12$. The value of $s$ is $s=0.0748830$, where $s \in(1 / 14,1 / 12)$, indicating that a $1 / 7$ periodic motion induced by grazing, as shown in Fig.5(c). (IV) $\zeta=0.107$. The value of $s$ is $s=0.0969813$, where $s \in(1 / 12,1 / 10)$, indicating that a $1 / 6$ periodic motion induced by grazing, as shown in Fig.5(d). (V) $\zeta=0.09$. The value of $s$ is $s=0.1152397$, where $s \in(1 / 10,1 / 8)$, indicating that a $1 / 5$ periodic motion induced by grazing, as shown in Fig.5(e).

(VI) $\zeta=0.068$. The value of $s$ is $s=0.1303988$, where $s \in(1 / 8,1 / 6)$, indicating that a 1/4 periodic motion induced by grazing, as shown in Fig.5(f).

Table 2 Eigenvalues, values of $s$ and the type of periodic orbits

\begin{tabular}{cccccc}
\hline Fig. Name & $\zeta$ & $\lambda_{1,2}$ & $s$ & Interval of $s$ & n-period orbits \\
\hline Fig. 5(a) & 0.1257 & $0.6265914 \pm 0.2476296 \mathrm{i}$ & 0.0598100 & $(1 / 18,1 / 16)$ & $1 / 9$-period orbit \\
Fig. 5(b) & 0.123 & $0.6189739 \pm 0.2803125 \mathrm{i}$ & 0.0676783 & $(1 / 16,1 / 14)$ & $1 / 8$-period orbit \\
Fig. 5(c) & 0.12 & $0.6113898 \pm 0.3109528 \mathrm{i}$ & 0.0748830 & $(1 / 14,1 / 12)$ & $1 / 7$-period orbit \\
Fig. 5(d) & 0.107 & $0.5859165 \pm 0.4089430 \mathrm{i}$ & 0.0969813 & $(1 / 12,1 / 10)$ & $1 / 6$-period orbit \\
Fig. 5(e) & 0.09 & $0.5646171 \pm 0.4992906 \mathrm{i}$ & 0.1152397 & $(1 / 10,1 / 8)$ & $1 / 5$-period orbit \\
Fig. 5(f) & 0.068 & $0.5513964 \pm 0.5901340 \mathrm{i}$ & 0.1303988 & $(1 / 8,1 / 6)$ & $1 / 4$-period orbit \\
\hline
\end{tabular}




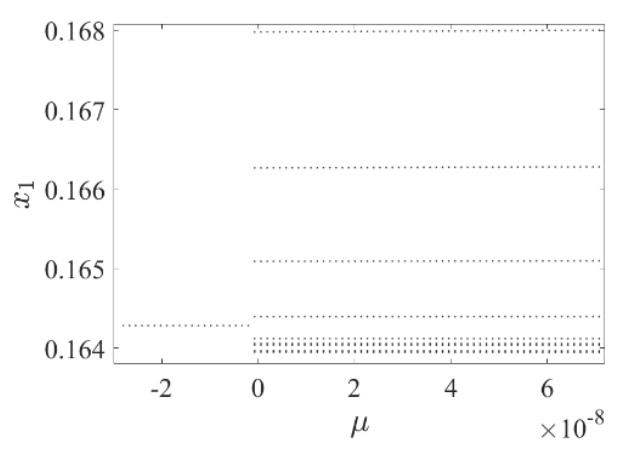

(a)

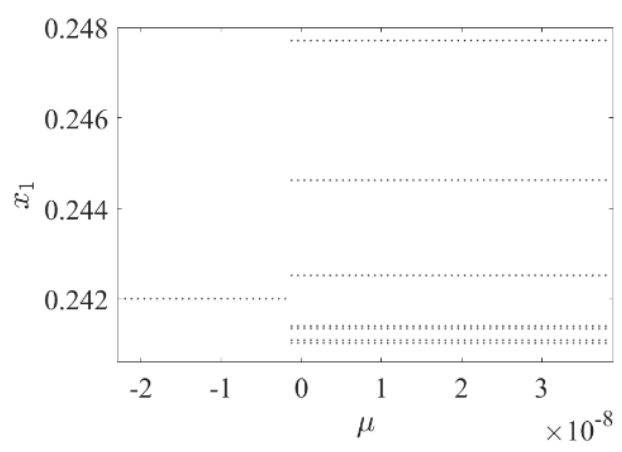

(c)



(e)



(b)

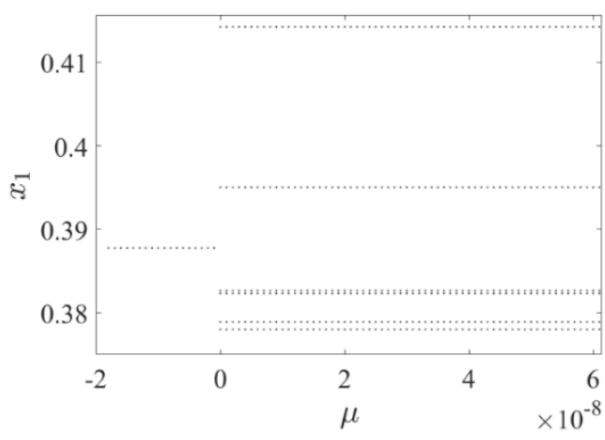

(d)

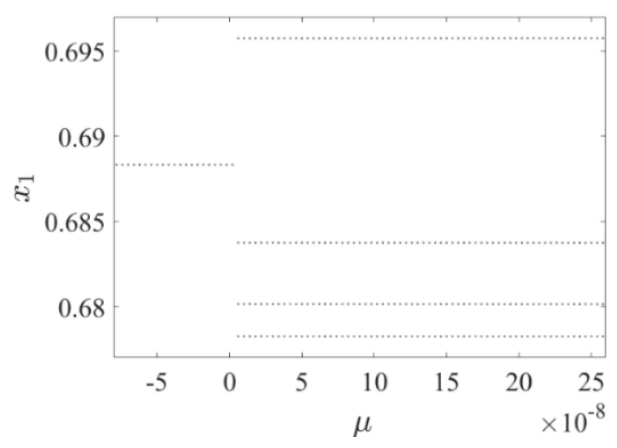

(f)

Fig. 5 The type of periodic orbits after grazing bifurcation in the case of conjugate complex eigenvalues. (a) $\zeta=0.1257: 1 / 9$-period-orbit (b) $\zeta=0.123: 1 / 8$-period orbit (c) $\zeta=0.12: 1 / 7$ period orbit (d) $\zeta=0.107: 1 / 6$-period orbit (e) $\zeta=0.09: 1 / 5$-period orbit (f) $\zeta=0.068: 1 / 4$ period orbit. 


\section{Conclusion}

Considering a cantilever beam system with a single impacting constraint, we uncover the existence and stability of periodic orbits induced by grazing. The non-smooth dynamic system is transformed into a combination of non-homogeneous equations and inequalities through a given impact mode, thereby avoiding the singularity of the local discontinuous mapping. Subsequently, we obtain the existence conditions and the stability criteria of the periodic orbits induced by grazing bifurcations. This stability criterion explains further the phenomenon that the low-period orbit can only transition to the high-period one as $\mu \rightarrow 0+$, which is known as period-adding bifurcation.

In the case that the eigenvalues are real numbers, we give sufficient conditions for the existence of $1 / n$ periodic motions. And, the conditions are further simplified into a simpler algebraic criterion by introducing the Jacobi matrix of the non-impact periodic orbit and its eigenvalues. Taking the impact clearance as the bifurcation parameter, the numerical simulation is used to verify the effectiveness of this criterion. It should be noted that in the case of $\lambda_{1}>2 / 3$, the numerical simulation shows that chaotic bands may alternate with periodic bands ,till the period-adding cascade appears when the parameter $\mu$ decrease and pass through some critical value.

In the case that the eigenvalues is a pair of complex numbers, we give a conjecture about the relationship between the $1 / n$ periodic motion and the eigenvalues based on a large number of numerical simulations. Ref.[24] gives a conjecture which $1 / n$ periodic orbit exists in the case of $0<s<1 / 2 n$. However, this paper show that the there is a $1 / n$-period motion induced by grazing in the case of $s \in(1 / 2 n, 1 / 2(n-1))$. The effectiveness of this conjecture remains to be proved theoretically in the future.

\section{Acknowledgments}

This work is supported by the National Natural Science Foundation of China (NNSFC) (Nos. 12072291, and 11732014). 


\section{Compliance with ethical standards}

Conflict of interest The authors declare that they have no conflict of interest.

\section{Data Availability Statements}

The datasets generated during and/or analysed during the current study are available from the corresponding author on reasonable request.

\section{References}

[1] Shaw S W, Holmes P J. A periodically forced piecewise linear oscillator[J]. Journal of Sound and Vibration, 1983, 90(1): 129-155.

[2] Whiston G S. Singularities in vibro-impact dynamics[J]. Journal of Sound and Vibration, 152(3), 1992: 427-460.

[3] Nordmark A B. Non-periodic motion caused by grazing incidence in an impact oscillator[J]. Journal of Sound Vibration, 145(2),1991: 279-297.

[4] Nordmark A B. Universal limit mapping in grazing bifurcations[J]. Physical Review E, 1997, 55(1): 266-270.

[5] Dankowicz H, Zhao X P. Local analysis of co-dimension-one and co-dimension-two grazing bifurcations in impact microactuators[J]. Physica D, 2005, 202(3-4): 238-257.

[6] Zhao X P. Discontinuity Mapping for Near-Grazing Dynamics in Vibro-Impact Oscillators[M]. Vibro-Impact Dynamics of Ocean Systems and Related Problems, Springer Berlin Heidelberg, 2009: 275-285

[7] di Bernardo M, Budd C J, Champneys A R. Normal form maps for grazing bifurcations in n-dimensional piecewise-smooth dynamical systems[J]. Physica D Nonlinear Phenomena, 2001, 160(3-4): 222-254.

[8] Li Q H, Wei L M, Tan J Y, et al. Double grazing periodic motions and bifurcations in a vibroimpact system with bilateral stops[J]. Abstract and Applied Analysis, 2014, 2014: 1-9.

[9] Zhang S J, Lu Q S, A non-smooth analysis to the rub-impacting rotor system[J]. Chinese Journal of Theoretical and Applied Mechanics, 2000, 32(1): 59-69.

[10] Feng J Q, X W, Grazing-induced chaostic crisis for periodic orbits in vibro-impact 
systems[J]. Acta Mechanica Sinica, 2013, 45(1):30-36.

[11] Luo G W, Xie J H, Sun X F, Periodic motions and global bifurcations of a two degree of freedom system with clearance[J]. Journal of the China Railway Society, 1999, 21(6): 24-29.

[12] Shen Y K, Yin S, Wen G L, et al. Linear feedback control of the discontinuous grazing bifurcation in a vibro-impact system[J]. Chinese Journal of Theoretical and Applied Mechanics[J], 2018, 35(4): 688-693.

[13] Shen Y K, Yin S, Wen G L, et al. Feedback control of grazing induced chaos in the singledegree-of-freedom impact oscillator[J]. Journal of Computational and Nonlinear Dynamics, 2018, 13(1): 011012.

[14] Simpson, D J W, Kuske, et al. The influence of localized randomness on regular grazing bifurcations with applications to impacting dynamics[J]. Journal of vibration and control, 2018, 24(2), 407-426.

[15] Simpson D J W, Avrutin V, Banerjee S. Nordmark map and the problem of large-amplitude chaos in impact oscillators[J]. Physical Review E, 2020, 102: 022211.

[16] Xu H D, Yin S, Wen G L, et al. Discrete-in-time feedback control of near-grazing dynamics in the two-degree-of-freedom vibro-impact system with a clearance[J]. Nonlinear Dynamics, 2017, 87(2):1127-1137.

[17] Jiang H B, Chong A S E, Ueda Y, et al. Grazing-induced bifurcations in impact oscillators with elastic and rigid constraints[J]. International Journal of Mechanical Sciences, 2017, 127: 204214.

[18] Brzeski P, Chong A S E, Wiercigroch M, et al. Impact adding bifurcation in an autonomous hybrid dynamical model of church bell[J]. Mechanical Systems and Signal Processing, 2018, 104: 716-724.

[19] Yin S, Wen G L, Wu X. Suppression of grazing-induced instability in single degree-offreedom impact oscillators[J]. Applied Mathematics and Mechanics, 2019, 40(1): 97-110.

[20] Yin S, Wen G L, Ji J C, et al. Novel two-parameter dynamics of impact oscillators near degenerate grazing points[J], International Journal of Non-Linear Mechanics, 2020, 120, 103403.

[21] Emans J, Wiercigroch M, Krivtsov A. Cumulative effect of structural nonlinearities: chaotic dynamics of cantilever beam system with impacts[J]. Chaos Solitons and Fractals, 2005, 
23(5): 1661-1670

[22] Wang L, Ni Q, Huang Y Y. Bifurcations and chaos in a forced cantilever system with impacts[J]. Journal of Sound and Vibration, 2006, 296(4-5): 1068-1078.

[23] Huangfu Y G, Li Q H. Grazing bifurcation of a vibrating cantilever system with one-side impact[J]. Chinese Journal of Theoretical and Applied Mechanics, 2008, 40(6): 812-819.

[24] Nordmark A B. Existence of periodic orbits in grazing bifurcations of impacting mechanical oscillators[J]. Nonlinearity, 2001, 14(6): 1517-1542.

[25] di Bernardo M, Budd C J, Champneys A R, et al. Piecewise-Smooth Dynamical Systems: Theory and Applications[M]. London: Springer-Verlag, 2008: 188-193. 